\title{
Neonatal Sepsis Past to Present
}

\author{
Santosh Kumar Kamalakannan* \\ Assistant professor of Neonatology, Saveetha medical college, India
}

Received: March 09, 2018; Published: April 04, 2018

*Corresponding author: Santosh Kumar Kamalakannan, Assistant professor of Neonatology Saveetha medical college, India, Tel: 9884980677;

Email: drsantoshkmc03@gmail.com

Abbreviations: EOS: Early Onset Neonatal Sepsis; LOS: Late onset Neonatal Sepsis; NEC: Necrotizing Enterocolitis; RDS: Respiratory Distress Syndrome; UTI: Urinary Tract Infection; GCSF: Granulocyte Colony-Stimulating Factor

\section{Introduction}

Neonatal sepsis is a clinical syndrome characterized by systemic signs of infection and bacteremia in the neonatal period [1]. Neonatal sepsis is a major cause of mortality and neurodevelopmental impairment among neonates. It contributes to nearly $30 \%$ of neonatal deaths in developing countries [2]. Inherent factors like poorly developed innate immune system, immature skin barrier, mucosal defense mechanisms and blood brain barrier contribute to the increased susceptibility of the neonates to infection .Early recognition and aggressive management plays a pivotal role in saving these neonates. Bacterial infections are the most common cause of septicemia in neonates but fungal and viral infections can occur in the setting of an extreme premature baby and by vertical transmission from the mother.

\section{Definition}

The term neonatal sepsis, refers to a clinical syndrome characterized by the blood stream infection of neonates and inflammatory response mounted by the neonate, it usually presents as septicemia, pneumonia, meningitis, and rarely as arthritis, osteomyelitis, and urinary- tract infection. Superficial infections like oral thrush and pustules are generally termed as local infections.

\section{Classification}

Based on the time of onset neonatal sepsis has been traditionally classified as [3].

a. Early onset Neonatal sepsis (EOS), defined as onset of manifestations is within 72 hours of birth.

b. Late onset Neonatal sepsis (LOS) is defined as onset of manifestations after 72 hours of life.
This classification helps in guiding the antibiotic therapy as it indicates the differences in the presumed mode of transmission and predominant organisms. EOS is mostly results due to vertical transmission of organisms from mothers to infants during the antepartum and intrapartum period and LOS is attributed to the horizontal transmission of pathogens acquired postnatally from the hospital environment or from the community.

\section{Vulnerability of the Neonate}

a. Immature innate immune system: Reduced phagocytic and opsonisation activity, low complement levels, and immature cell mediated and humoral immunity.

b. Poor barrier to infection: Fragile immature skin easily susceptible to invasion of organism, immature mucosal barriers and reduced levels if secretory immunoglobulins ,immature ciliary function with reduced ability to clear secretions and poorly developed blood brain barrier.

\section{Risk Factors}

\section{Early onset Neonatal sepsis (EOS):}

Presence of the following risk factors has been associated with an increased risk of EOS [3,4].

a. Low birth weight $(<2500 \mathrm{gms})$ or prematurity.

b. Febrile illness in the mother 2 weeks prior to delivery.

c. Foul smelling and/or meconium stained liquor.

d. Prolonged rupture of membrane ( $>24$ hours).

e. More than 3 vaginal examinations during labor.

f. Prolonged and difficult delivery with instrumentation. 
Perinatal asphyxia (Apgar score $<4$ at 1 minute of age) or difficult resuscitation. Neonates with presence of foul smelling liquor or any three of the above mentioned risk factors should be considered to have EOS \& started on antibiotics. Neonates with $\geq 2$ risk factors should be investigated for sepsis with sepsis screen and treated accordingly.

\section{Late onset Neonatal sepsis (LOS)}

Factors associated with the increased risk of LOS in hospital setting includes poor hand hygiene ,low birth weight ,prematurity, invasive ventilation, use of parenteral nutrition, prolong use of antibiotics ,invasive central lines and intravenous catheters [5,6]. Factors associated with community acquired late onset sepsis includes, use of prelacteal feeds, artificial feeds, bottle feeding, and unhygenic cord and skin care $[5,6]$. Breastfeeding has been found to offer protection against the development of late onset sepsis [7].

\section{Causative Organism}

The organisms implicated in the development of neonatal sepsis vary from place to place. In developed countries Group B streptococcus and Coagulase negative staphylococcus are the major organisms implicated in early onset and late onset sepsis respectively [8]. In India gram negative organisms predominate in both early and late onset sepsis. In the recently conducted DeNISstudy, the most frequently isolated organisms among inborn cohort were Acinetobacter spp followed by Klebsiella. Among outborn cohort Klebsiella was the most common pathogen isolated. Fungal isolates were responsible for one -fourth of systemic infections [9].

\section{Clinical Presentation}

Neonatal sepsis presents with non specific clinical symptoms and signs. Manifestations of sepsis are very difficult to differentiate from many other conditions which can present in fashion. In the presence of risk factors clinician must rule out sepsis by additional diagnostic evaluation. EOS usually presents as respiratory distress soon after birth. In the instance of fulminant infection intrauterine manifestations like fetal tachycardia, reduced fetal movements or excessive fetal movements are observed. Usual presentations of late onset sepsis include generalized septicemia, pneumonia, and meningitis. Rarely septic arthritis and osteomyelitis can be manifestation of late onset sepsis. A high index of suspicion and vigilant clinical assessment is essential for early identification of Neonatal sepsis. System specific manifestation are identifiable in an around 30 to $50 \%$ of the cases and should be looked for in all cases of suspected neonatal sepsis.

\section{Non-Specific Manifestations of Neonatal Sepsis}

a. Poor feeding or unable to feed.

b. Lethargy, poor cry and coma.

c. Hypothermia or fever (former is more common in LBW babies).

d. Apnea or gasping respiration and cyanosis. e. Bradycardia or tachycardia, Poor perfusion i.e. Prolonged capillary refill time ( $>2$ seconds), Hypotension and shock.

f. Jaundice.

g. Hypotonic or absent neonatal reflexes.

h. Oliguria or Anuria.

i. Acidotic breathing.

\section{System Specific Manifestation}

Respiratory System: Tachypnea, Chest retractions, grunt, alar nasi flare and cyanosis these signs are indicative of Pneumonia. Gastrointestinal: Feed intolerance, vomiting, diarrhea, abdominal distension and sluggish or absent bowel sounds. Presence of these signs in a preterm infant may denote a high possibility of necrotizing enterocolitis.

Central Nervous System: Bulging anterior fontanel, blank look, high-pitched cry, excessive Irritability, coma, seizures, and neck retraction. Presence of these signs should raise clinical suspicion of meningitis.

Skin: There may be multiple pustules, large abscess, mottling of skin, purpura and sclerema. Diagnostic workup: Isolation of microorganisms from blood, CSF, urine or other body fluids is diagnostic of sepsis. As a sick neonate would require early initiation of antimicrobial therapy workup should be rapid and specific [10]. Blood Culture: Blood culture should be done in all suspected cases before initiating antimicrobial therapy. Fresh venipuncture should be performed after proper skin preparation by using $70 \%$ isopropyl alcohol and povidone-iodine. The skin should be allowed to dry for at least 1 minute before the sample collection. Blood samples collected from indwelling catheters or lines are likely to be contaminated and should be avoided. The volume of blood inoculated and amount of culture medium used for culture plays an important role in the yield of blood culture. Usually one-mL of blood should be inoculated for a blood culture bottle containing 5-10 mL of culture medium. Recently introduced BACTEC or BACT/ ALERT culture system can detect bacterial growth within 12-24 hours.

\section{Sepsis Screen}

There are number of blood investigations other than culture used to detect sepsis. The commonly used septic screen consists of Total leucocyte count, Absolute neutrophil count, I: T ratio, micro ESR and CRP [11,12]. However, the decision to start antibiotics should not be dictated by the sepsis screen result, if there is a strong clinical suspicion of sepsis antimicrobial therapy should be started. Sepsis screen is considered positive if two of the parameters are positive. If the screen is negative but clinical suspicion persists, it should be repeated within 12 hours. If the screen is still negative, sepsis can be excluded with reasonable certainty. A positive septic screen however may not be confirmatory as other clinical conditions like PIH, Perinatal asphyxia, shock can alter the results of the parameters and result in a false positive screen. 
The parameters tested as a part of septic screen include: $[11,12]$ (Table 1).

Table 1: Septic Screen.

\begin{tabular}{|c|c|}
\hline Component & Abnormal value \\
\hline TLC & $<5000 / \mathrm{mm} 3$ \\
\hline ANC & $\begin{array}{c}<\text { as per Manroe chart for term. } \\
\text { Mouzinho's chart for VLBW } \\
\text { infants }\end{array}$ \\
\hline $\begin{array}{c}\text { Immature/total neutrophil (I:T } \\
\text { RATIO) }\end{array}$ & $>0.2$ \\
\hline Micro-ESR & $>15 \mathrm{~mm} \mathrm{in} 1 \mathrm{st} \mathrm{hr}$ \\
\hline CRP & $>1 \mathrm{mg} / \mathrm{dl}$ \\
\hline
\end{tabular}

\section{Lumbar puncture (LP)}

Meningitis can be the presentation of neonatal sepsis in around $3 \%$ of the cases and hence CSF analysis should be contemplated in all neonates with late onset sepsis prior to starting antibiotics [13]. In EOS, lumbar puncture is indicated in the presence of a positive blood culture, or when the clinical picture is consistent with septicemia. Lumbar puncture may be deferred in the following situations: [14]

a. Asymptomatic babies being investigated for maternal risk factors; However, LP should be done in these cases, if blood culture becomes positive, subsequently.

b. Premature neonates afflicted with respiratory distress syndrome (RDS); In this case, LP should be postponed in critically-ill and haemodynamically-unstable babies (Table 2).

Table 2: Normal CSF findings in Neonatal Sepsis.

\begin{tabular}{|c|c|c|}
\hline Tests in CSF & Term & Preterm \\
\hline Cells & $7(0-32)$ & $9(0-29)$ \\
\hline Polymorphonuclear cells & $61 \%$ & $57 \%$ \\
\hline Protein(mg/dl) & $90(20-170)$ & $115(65-150)$ \\
\hline Glucose $(\mathrm{mg} / \mathrm{dl})$ & $52(34-119)$ & $50(24-63)$ \\
\hline Protein:glucose & $81(44-288)$ & $74(55-105)$ \\
\hline
\end{tabular}

c. Pleocytosis, elevated protein levels, low glucose levels are features suggestive of meningitis. Corresponding blood glucose should be done alone with CSF glucose for comparision.

\section{Radiology}

Chest x-ray should be done in presence of respiratory distress and abdominal distention. Classical features of sepsis in Chest x-ray include a hyper inflated lung fields, tented diaphragm, dilated bowel loops and apatch infiltration in the lung field in case of a bronchopneumonia. An x-ray and ultrasound of abdomen should be done in the presence of abdominal signs suggestive of necrotizing enterocolitis (NEC) or paralytic ileus or blood in stools. Neurosonogram and CT brain should be performed in all neonates diagnosed or suspected to havemeningitis. Extra-axial fluid collection, echogenic widening of the brain sulci, meningeal thickening and hyperemia suggest a diagnosis of meningitis in Neurosonogram. Intraventricular debris and stranding, and an irregular and echogenic ependyma are highly suggestive findings associated with ventriculitis.

\section{Urine culture}

The rate of positive urine culture in infants with early onset sepsis is very low and hence urine culture should not be part of traditional sepsis work up in the first 72 hours of life. Urine cultures obtained by suprapubic puncture or bladder catheterization have been recommended in all cases of LOS. Neonates at risk for fungal sepsis and very low birth weight infants with poor weight gain and those with suspected urogenital malformation should have a urine examination done to exclude urinary tract infection (UTI).

UTI may be diagnosed in the presence of one of the following: [15].

a. $\quad>10 \mathrm{WBC} / \mathrm{mm}$ in a $10 \mathrm{~mL}$ centrifuged sample.

b. $>10$ organisms / $\mathrm{mL}$ in urine obtained by catheterization.

c. Any organism in urine obtained by suprapubic aspiration.

\section{Additional Investigations to be Considered:}

a. Blood gas, renal function tests, liver function and coagulation profile.

b. Maternal high vaginal swabs in case of EOS. Viral and fungal study in appropriate situations.

\section{Newer Diagnostic Test:}

Recently new diagnostic modalities have been studied for early identification of sepsis .These Diagnostic agents is broadly classified as [16].

Acute Phase Reactants: These groups of endogenous peptides are produced by the liver as part of an immediate response to the infection or tissue injury. These reactants are C-reactive protein, procalcitonin, fibronectin, haptoglobin, lactoferrin, neopterinand oromucosoid. Of these CRP and Procalcitonin have shown promising results.

Cell Surface Markers: Neutrophil CD11b and CD64 appear to be promising markers for the diagnosis of early- and late-onset infections. Other markers that have been studied include CD33, CD66b, and CD19 .These markers are induced by inflammation secondary to bacterial infections.

Granulocyte colony-stimulating factor: Granulocyte colonystimulating factor (GCSF), a mediator produced by bone marrow, facilitates proliferation and differentiation of neutrophils, and has been proposed to be a reliable infection marker for early diagnosis of neonatal sepsis.

Cytokines: TNF-a, IL-1b, IL-6, IL-8, p55, p75, and IL-1 receptor antagonist have been studied in predicting neonatal sepsis. Of these markers IL-1b, IL-6, and IL-8 are more useful clinical setting and have been studied extensively. Umbilical cord IL- 6 levels have been found to be a good marker in early diagnosis of EOS. 
Molecular Genetic Tests: Molecular genetics techniques can help the treating neonatologist in the diagnosis of neonatal sepsis by identifying specific fungal, bacterial and viral genes in neonatal blood through amplification of target DNA/RNA fragments. 16S r RNA is the most widely evaluated of these RNA fragments.

\section{Management}

Management of a neonate with sepsis includes providing aggressive supportive care, antimicrobial therapy and adjuvant therapies.

\section{Supportive Care}

a. Maintenance of thermo-neutral environment, prevention of hypo or hyperthermia.

b. Maintenance of normoglycemic status (45 to $120 \mathrm{mg} / \mathrm{dl}$ ).

c. Maintenance of Oxygen saturation (91 to $94 \%$ ).

d. Maintenance of tissue perfusion and blood pressure using colloids and inotropes.

e. Maintenance of adequate nutrition by enteral feeding if not feasible by parenteral nutrition.

f. Blood products to normalize the coagulation abnormalities, correction of anemia and thrombocytopenia.

\section{Antimicrobial Therapy}

The choice of antimicrobial drug for an infant with suspected sepsis depends upon the predominant pathogen and antibiotic sensitivity pattern of the given hospital and hence there cannot be a universal recommendation that can be used in all settings. The decision to initiate antibiotic therapy depends on the results of septic screen and the clinical findings present in the baby, the following the major indications for stating antibiotic therapy.

\section{Indications for Starting Antibiotics}

The indications for starting antibiotics in neonates at risk of EOS include:
a. Presence of $>3$ risk factors for early onset sepsis.
b. Presence of foul smelling liquor.
c. presence of 2 antenatal risk factor and a positive septic screen and '
d. Strong clinical suspicion of sepsis.

\section{The indications for Starting Antibiotics in LOS Include}

a. Positive septic screen.

b. Strong clinical suspicion of sepsis.

There is generally no role for the use of prophylactic antibiotics in preterm babies and those babies on intravenous fluids or parenteral nutrition, birth asphyxiated babies (intrapartum asphyxia).However prophylactic antibiotics may be considered in sick neonates requiring prolonged ventilation.

\section{Antibiotic Therapy}

a. In neonates with suspected community-acquired sepsis, resistant strains are unlikely, and hence a combination of ampicillin and gentamicin may be started for septicemia and pneumonia .A third-generation cephalosporin e.g. cefotaxime, is added if meningitis is present in these cases. Cefotaxime should be used because of its reduced toxicity and broad spectrum of coverage. Ceftriaxone is generally not preferred due to its potential to displace bilirubin from albumin, and hence increasing the risk of jaundice and bleeding.

b. In the setting of hospital-acquired sepsis, there is a high probability of resistant strains, cefotaxime in combination with an aminoglycoside, should be used for septicemia and pneumonia, as well as for meningitis.

c. In units with high incidence of resistance piperacillintazobactam or methicillin/vancomycin are preferred.

d. A combination of piperacillin/tazobactam with amikacin should be considered if Pseudomonas sepsis is suspected.

Empiric antifungal therapy should be considered if the infants have central vascular ccess, an endotracheal tube, and thrombocytopenia, exposure to broad spectrum cephalosporin's or carbapenem, and gestational age less than 28 weeks. Amphotericin B should be chosen for empiric therapy, and fluconazole should be reserved for prophylaxis.

\section{Duration of Antibiotic Therapy}

a. Clinical sepsis (Based on clinical suspicion and/or sepsis screen positivity):7-10 days.

b. Culture positive sepsis (not meningitis), UTI -14 days.

c. Meningitis-2 weeks after sterilization of CSF culture or for a minimum of 2 weeks for gram positive meningitis and 3 weeks for gram negative meningitis, whichever is longer.

d. Bone and joint infection-4-6 weeks.

\section{Upgrading Antibiotics}

Upgrading of antibiotics may be considered if there is no clinical improvement by 48 hours of starting the antibiotics or if there is further clinical deterioration. Antifungal therapy should be considered if the infants have central vascular access, thrombocytopenia, exposure to prolongedantibiotics, in gestational age less than 28 weeks. Fluconazole and amphotericin B are commonly used antifungal agents.

\section{Adjuvant Therapies}

Interavenous Immunoglobulin: There is little evidence to suggest the use of nonspecific IVIG for the treatment of suspected or proven neonatal sepsis [17].

Exchange Transfusion: It may be used with caution in neonatal sepsis associated with neutropenia, sclerema, disseminated intravascular coagulation, and metabolic acidosis ( $\mathrm{pH}<7.2)$ [18]. 
Granulocyte Colony Stimulating Factor: There is currently insufficient evidence to support the introduction of either G-CSF or GM-CSF into neonatal practice for management of sepsis [19]. Other Therapies: Melatonin, zinc, pentoxyfyllin, probiotics has been studied with inconsistent results .Of these probiotics seems to be the most useful at present (Table 3).

Table 3: Commonly used Drugs in Neonatal Sepsis.

\begin{tabular}{|c|c|c|c|}
\hline \multirow{2}{*}{ Drug } & \multirow{2}{*}{ Dose } & \multicolumn{2}{|c|}{ Frequency* } \\
\cline { 3 - 4 } & & $<\mathbf{7}$ days & $>\mathbf{7}$ days \\
\hline Ampicillin & $50 \mathrm{mg} / \mathrm{kg} /$ dose & BD & TDS \\
\hline Amikacin & $15 \mathrm{mg} / \mathrm{kg} /$ dose & OD & OD \\
\hline Gentamicin & $5 \mathrm{mg} / \mathrm{kg} /$ dose & OD & OD \\
\hline $\begin{array}{c}\text { Piperacillin } \\
\text { tazobactum }\end{array}$ & $50 \mathrm{mg} / \mathrm{kg} /$ dose & BD & TDS \\
\hline Ciprofloxacin & $10 \mathrm{mg} / \mathrm{kg} /$ dose & BD & BD \\
\hline Vancomycin & $10 \mathrm{mg} / \mathrm{kg} /$ dose & OD & OD \\
\hline Meropenam & $20 \mathrm{mg} / \mathrm{kg} /$ dose & TDS & TDS \\
\hline Cefotaxime & $50 \mathrm{mg} / \mathrm{kg} /$ dose & BD & TDS \\
\hline
\end{tabular}

\section{Prevention}

\section{Antenatal Period}

a. Regular Antenatal visits and early identification and treatment of urinary tract infection.

b. Immunization with tetanus toxoid and influenza vaccines.

13.2. Intrapartum Period

a. Strict asepsis and handwashing during deliveries.

b. Chlorhexidine wipes use in mother's genital tract before delivery.

c. Intrapartum antibiotic prophylaxis.

\section{Postnatal Period}

a. Chlorhexidine wiping of the neonates daily.

b. Strict hand washing of all care takers.

c. Exclusive breastfeeding: Breast milk contains secretory IgA, lysozymes, white blood cells, and lactoferrin, and has been shown to encourage the growth of healthy lactobacilli and reduce the growth of E. coli and other Gram-negative pathogenic bacteria. Early initiation and exclusive breastfeeding rates demonstrated significant reductions in diarrhea and acute respiratory infections in neonates and older infants in India.

d. Immunization: Nonspecific effects of BCG has been studied in the BLOW trial which has shown that BCG administration in low birth weight neonates was associated reduced mortality and sepsis. e. Micronutrient supplementation: Use of Vitamin A has been shown to reduce incidence of BPD and hence indirectly reduce pneumonia episodes.

\section{References}

1. Klein JO (1990) Bacteriology of neonatal sepsis.The Pediatric Infectious Disease Journal 9(10): 777-7778.

2. Bang AT, Bang RA, Bactule SB, Reddy HM, Deshmukh MD (1999) Effect of home-based neonatal care and management of sepsis on neonatal mortality: field trial in rural India. Lancet 354(9194): 1955-1961.

3. Singh M, Narang A, Bhakoo ON (1994) Predictive perinatal score in the diagnosis of neonatal sepsis.J Trop Pediatr 40(6): 365-368.

4. Takkar VP, Bhakoo ON, Narang A (1974) Scoring system for the prediction of early neonatal infections. Indian Pediatr 11(9): 597-600.

5. Baltimore RS (1998) Neonatal nosocomial infections. Semin Perinatol 22(1): 25-32.

6. Wolach B (1997) Neonatal sepsis: pathogenesis and supportive therapy. Semin Perinatol 21(1): 28-38.

7. Ashraf R, Jalil FE, Zaman SH, Karlberg J, Khan SR, et al. (1991) Breast feeding and protection against neonatal sepsis in a high risk population. Archives of disease in childhood 66(4): 488-490.

8. Camacho-Gonzalez A, Spearman PW, Stoll BJ (2013) Neonatal infectious diseases: evaluation of neonatal sepsis. Pediatr Clin North Am 60: 367389.

9. Agarwal R, Sankar J (2016) Characterisation and antimicrobial resistance of sepsis pathogens in neonates born in tertiary care centres in Delhi, India: a cohort study. The Lancet Global Health 4(10): e752-760.

10. Gerdes JS, Polin R (1998) early diagnosis and treatment of neonatal sepsis. Indian J Pediatr 65(1): 63-78.

11. Polinski C (1996) The value of white blood cell count and differential in the prediction of neonatal sepsis. Neonatal Netw 15(7): 13-23.

12. Da Silva O, Ohlsson A, Kenyon C (1995) Accuracy of leukocyte indices and C-reactive protein for diagnosis of neonatal sepsis: a critical review. Pediatr Infect Dis J 14(5): 362-366.

13. (2005) Report of the National Neonatal PerinatalDatabase. Report 2002-2003. NNPD Network.

14. Paul V, Agrawal R Neonatal sepsis. In NNF Manual of Neonatal Care. $1^{\text {st }}$ (Edn.). Prism Books Pvt. Ltd, Bangalore, India, pp. 121-34.

15. Richard A, Polin, Elvira P (2005) In Bacterial sepsis and meningitis. William T, Roberta A, Christine A (Eds.) very's Disease of the Newborn, $8^{\text {th }}(E d n$.$) . pp. 551-577.$

16. Kale A, Bonde V (2014) Neonatal Sepsis: An Update. Iranian Journal of Neonatology IJN 4(4): 39-51.

17. INIS Collaborative Group, Brocklehurst P, Farrell B, King A, Juszczak E, et al. (2011) Treatment of neonatal sepsis with intravenous immune globulin. New England Journal of Medicine 365(13): 1201-1211.

18. Sadana S, Mathur NB, Thakur A (1997) Exchange transfusion in septic neonates with sclerema: effect on immunoglobulin and complement levels. Indian Pediatr 34(1): 20-25.

19. Carr R, Modi N, Doré CJ (2003) G-CSF and GM-CSF for treating or preventing neonatal infections. Cochrane Database Syst Rev 3: CD003066. 
(c) (i) This work is licensed under Creative Submission Link: https://biomedres.us/submit-manuscript.php

\begin{tabular}{ll} 
BIOMEDICAL & Assets of Publishing with us \\
RESEARCHES & - Global archiving of articles \\
\hline ISSN:2574-1241 & - Immediate, unrestricted online access \\
\hline
\end{tabular}

